\title{
Using Environment Exploration for Inverse Kinematics Learning *
}

\author{
Wandemberg Gibaut* Ricardo Gudwin* \\ * DCA-FEEC-UNICAMP, Av. Albert Einstein 400, 13083-852, \\ Campinas, SP, (e-mails: wgibaut@dca.fee.unicamp.br and \\ gudwin@unicamp.br).
}

\begin{abstract}
Psychological theories about how environment exploration impacts on one's learning, specially in early ages, have great influence in areas as Humanoid Robotics and Cognitive Systems. In this paper we propose an approach based on the idea of autonomous exploration to shape behaviors of an agent, in order to learn the inverse kinematics of a 6-DOF robotic arm.
\end{abstract}

Keywords: Artificial Intelligence; Autonomous Agents; Cognitive Systems; Robotics; Neural Networks.

\section{INTRODUCTION}

The research areas of humanoid robotics and cognitive systems took a lot of inspiration in biologic and human sciences, like psychology and learning theories. The impact of learning processes on the sensory-motor development, specially in early ages of human children are an important source of inspiration for the creation of models of artificial agents embedded in its environment.

Despite some of our basic behaviors seem to be "hard coded" by evolution, these are not rigid mechanisms that can't change. In fact, most of them might be of little or no use if not further developed. Regarding this development, emerging action capabilities are heavily shaped by the agent interaction with the environment where it's embedded (Vernon et al., 2011). Also, in humans, the first few months are those where the nervous system develops more intensely. In this period, there is a huge generation of new synaptic connections on the cerebral cortex (Huttenlocher, 1990).

According to Gibson (1988), knowledge and representations of the world must be grounded in information somehow obtained from it. Even more, as living creatures, we need to deal with the environment, binding abstract concepts to more concrete and affordance-oriented ones. So, an exploration of the surrounding environment is almost mandatory. Yet according to Gibson (1988), this knowledge acquired from exploratory activities is probably entirely utilitarian in the beginning, simply focused on interactions that may reoccur, but constitutes a fundamental step to the ability to make predictions about the world.

With this in mind, we proposed a cognitive controller using these theories to present a solution to the inverse kinematics problem of a robotic arm, that is, to find

\footnotetext{
* This study was financed in part by the Coordenação de Aperfeiçoamento de Pessoal de Nível Superior - Brasil (CAPES) - Finance Code 001. The authors also thank Ericsson Research Brazil, Ericsson Telecomunicações S.A. Brazil (Proc. FUNCAMP 4881.7) and CEPID/BRAINN (Proc. FAPESP 2013/07559-3) for supporting this research.
}

a configuration of joints positions that brings the arm extremity to a desired point of the space, with a given orientation.

In the literature, this issue of motor exploration and learning is addressed in many related efforts. For example, Ivaldi et al. (2014) works with object properties, affordances and manipulation learning. There are also other approaches addressing the inverse kinematics problem (Wang and Chen, 1991; D'Souza et al., 2001).

\subsection{Cognitive Architectures}

The research field of Cognitive Architectures emerged as an offspring of Artificial Intelligence in the early 1990s, starting from a background in rule-based systems and symbolic computation (Newell and Simon, 1961; Simon and Newell, 1971). Based on his theory for the origins of human knowledge, Anderson (1989) developed ACT$\mathrm{R}$, one of the first cognitive architectures (Anderson et al., 2004; Anderson, 2009), more or less at the same time that Laird released the first versions of SOAR (Laird, 2012), another cognitive architecture based on the ideas of Newell et al. (1989). In the late 1990s a group of researchers working in the field of Simulation of Adaptive Behavior coined the concept of Cognitive Architecture as an essential set of structures and processes necessary for the generation of a computational-cognitive model, which can be used in several areas relating cognition and behavior. Sloman (2002), for example, addressed the "architectural" question to represent mental concepts, and Sun (2004) described some requirements needed to construct a Cognitive Architecture. Sun (2007) further addressed issues and challenges in the development of such models. Langley et al. (2009) analyzed many of these issues, evaluating the progress in the field. These studies indicated that the main advantage of characterizing an architecture as cognitive was the possibility of obtaining a concrete framework for cognitive modeling such that these models might be tested consistently. Such characterization would allow the definition of a set of basic structures, modules and processes, 


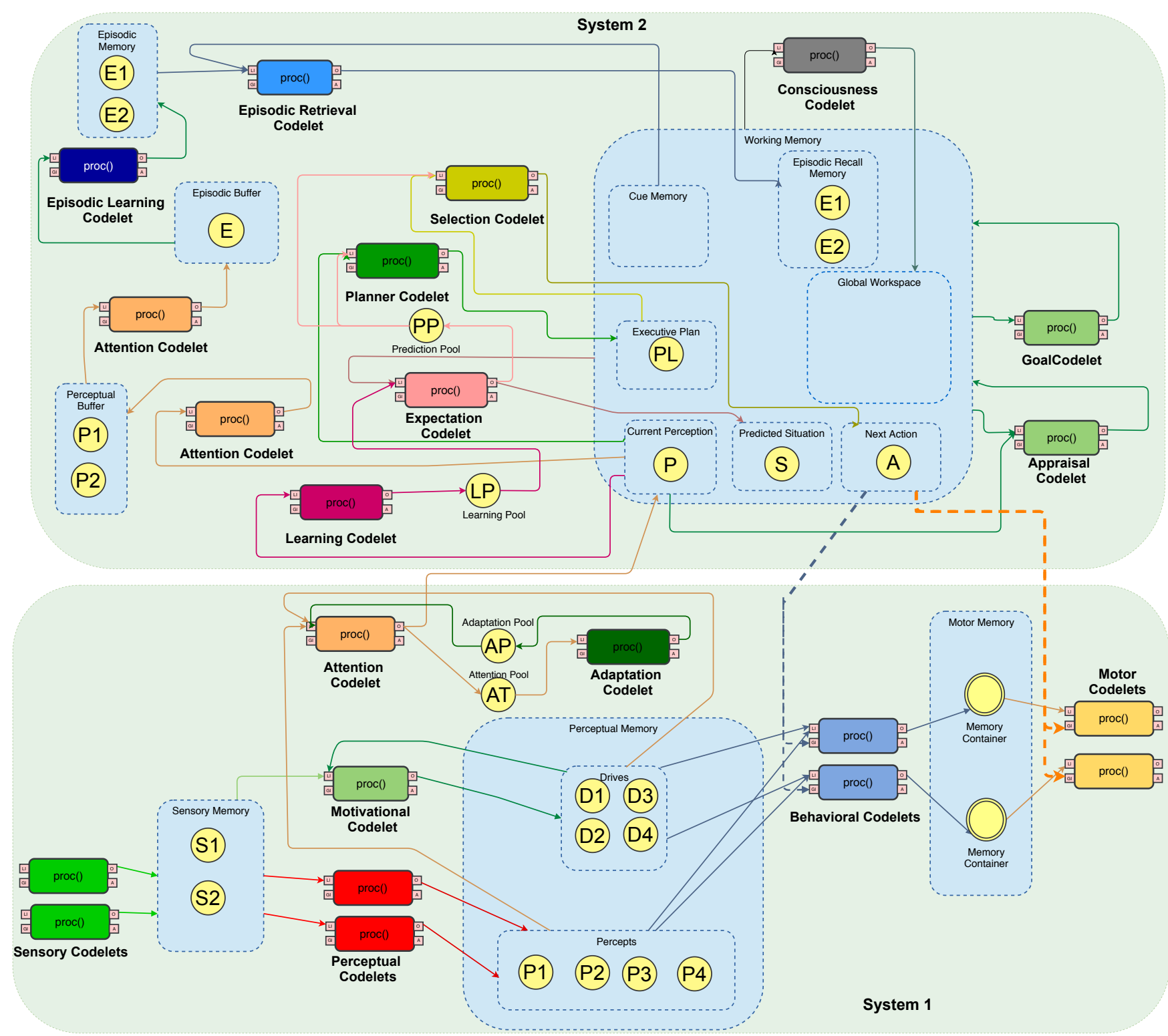

Figure 1. Overview of the modified, learning-focused, MECA architecture as seen in Gibaut (2018).

creating a common language such that different researchers might explore their proposals of cognitive models.

The field of Cognitive Architectures has many recent contributions, basically from two communities dealing with the issues of AGI-Artificial General Intelligence and BICA - Biologically Inspired Cognitive Architectures. In 2016, Kotseruba et al. (2016) presented a review covering the last 40 years of research in Cognitive Architectures.

\subsection{The Multipurpose Enhanced Cognitive Architecture (MECA)}

The MECA (Gudwin et al., 2017) Cognitive Architecture was developed considering many ideas coming from Dual Process Theory (Osman, 2004), Dynamic Subsumption (Arkin et al., 1998), Conceptual Spaces (Gärdenfors, 2014) and Grounded Cognition (Barsalou, 2010) and is built using the CST (Paraense et al., 2016) toolkit developed by our research group, which allows an user to create his own cognitive architecture.
First of all, MECA is an implementation of Dual Process Theory (Osman, 2004), i.e., MECA is divided into two large subsystems, called System 1 and System 2, which are independent and complementary in the construction of behavior. According to Dual Process Theory, System 1 is responsible for the fast, unconscious part of the cognitive process. It is mainly composed by reactive behaviors, but also might includes motivational behaviors, as e.g. "instincts". It contains 3 types of memory: Sensory Memory, Perceptual Memory and Motor Memory. These memories are accessed by various subsystems such as the behavioral, perceptual, and motivational subsystems in System 1.

The basic design of System 1 is a dynamic subsumption architecture. The inputs to this mechanism can come directly from the sensory memory, but they usually undergo some kind of perceptual treatment, that is, the generation of Percepts structures, which correspond to abstractions of the sensory data, that can be used in other parts of MECA. In particular, in System 1, those Percepts are used 


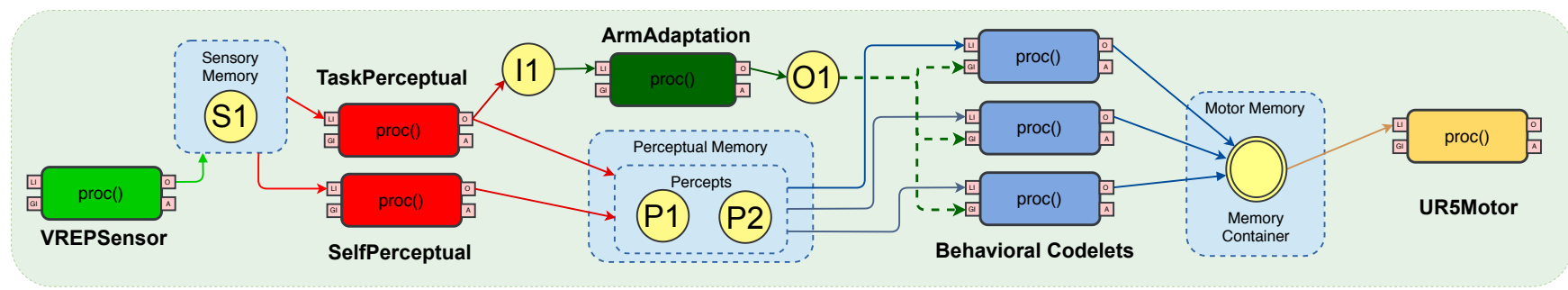

Figure 2. Topology of the controller built using the MECA architecture. The colors of the codelets follows those on figure 1 to easy association.

by behavioral Codelets. Among these Codelets, there is a special subgroup, the Motivational Behavior Codelets, which integrate the motivational subsystem of System 1 into them. The implementation details of System 1 can be found in (Gudwin et al., 2017).

System 2 is responsible for the slow, conscious process, as e.g. deliberative thinking. It is driven by a sequential rule-based process, operating with symbols and taking in account not just the present, but also the past and the future. It is in this system that planning processes take place, responsible for simulating the future and executing action plans aiming at high-level goals, and imagination, used as an aid to test possible courses of action and evaluate their pertinence. System 2 has, in its structure, higher-level subsystems, such as planning, expectation and motivation of System 2. It is also in this System that, through the Expectations subsystem, attempts are made to forecast the future in the short term and to learn from possible divergences between the expected and the reached. The specification details of this system can also be found in (Gudwin et al., 2017; Gibaut, 2018).

Although the original version of MECA was proposed by Gudwin et al. (2017), further developments on its structure were proposed later by Gibaut (2018), mainly addressing the issue of learning, not explicitly considered in the original architecture. Figure 1 provides an overview of the modified topology of the architecture. This version proposed many new codelets to address the issue of learning, specially in System 2. In this work, though, we focus on System 1.

\section{THE CONTROLLER}

Using the MECA architecture, we built a cognitive controller to the specific problem of inverse kinematics, shown in figure 2. Note that, despite MECA's dual process approach, this controller was made using only System 1. According to the theory, this would represent simple minds that lack the higher cognitive functions.

The color scheme used in the figure 2 follows that used in figure 1 , in order to become easy to identify the purpose of each codelet in the controller.

The VrepSensor present in figure 2 represents the controller's only sensory codelet. This codelet is responsible for getting information from the virtual environment and passing it, as a raw information, to the Perceptual Codelets. In the experiments regarding this paper, this codelet gets information about the joints position (a 6 - element double array), connector position and orientation (both 3-element double arrays) and the simulation time (a double value).

The two Perceptual Codelets seen in figure 2 are called SelfPerceptual and TaskPeceptual. As their names suggest, they are responsible, respectively, for the information processing regarding the agent itself and the tasks they should do. The first one stores new information about both the connector pose (position plus orientation) and the joints position, passing them to the behavioral codelets, as they reach a certain size: 50 entries each here, the same number of generated tasks. The other one is responsible for generating a list of target points (tasks) within the arm workspace, shown in figure 3 . This codelet is also responsible for constantly checking if the task was accomplished and passing information to the behavioral codelets regarding the current task and about the activation turn, discussed in section 2.1 .

All the Behavioral Codelets present in figure 2 are instances of the same class: (BasicBehavior), differing only in their internal neural networks structure. These codelets use information passed by the TaskPerceptual codelet as input to their neural networks to generate a 6-element double array that represents the instructions to be passed to the arm's joints, through the motor codelet. Also, when

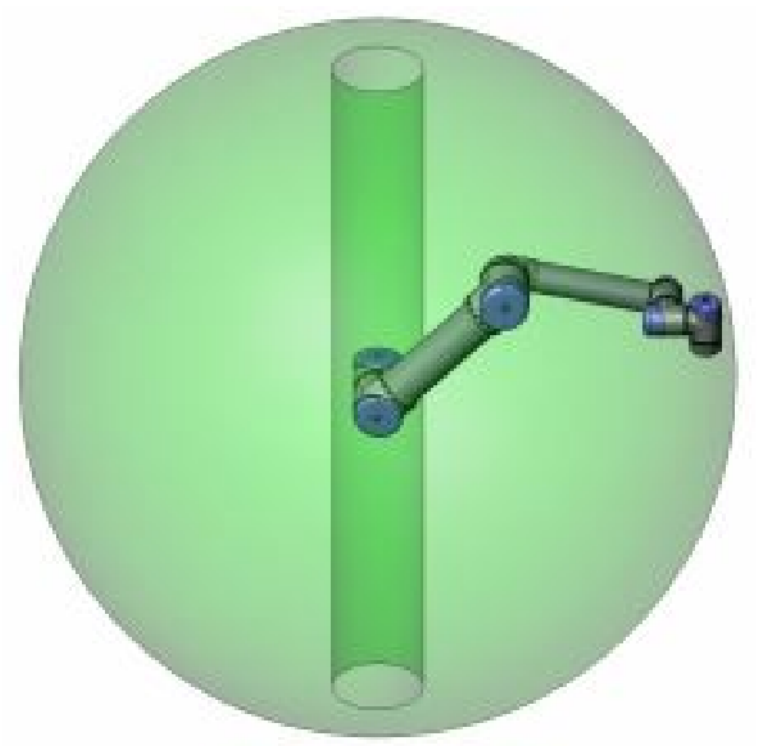

Figure 3. UR5 workspace: a 1 meter radius sphere excluding a cylinder centered on the first joint. Extracted from de Gier (2015). 
get info from the SelfPerceptual, they use it to train their networks, to achieve better results. Regarding the networks, they are all MLPs (MultiLayer Perceptrons) with 2 hidden layers. The number of neurons in each layer are passed when the instance is called and, in this problem, must have 6 neurons both in the input and the output, as the proposed solution states. The networks have also the following relevant information:

- Weight Initialization: Xavier

- Optimization Algorithm: Stochastic Gradient Descent

- Learning Rate: 0.01

- Updater: adaGrad

- Activation Function: TanH, except on the output layer, which is Identity

- Loss Function: L2 Loss

The package used to build the network was the Deep Learning $4 j(\mathrm{dl} 4 \mathrm{j})$, a versatile yet powerful tool to make neural networks in java.

In section 3 we bring more information about the networks training.

Following, we have the UR5Motor, the only Motor Codelet. Its function is only to get instructions from the Behavioral Codelets, passing it to the environment. So the VrepSensor and the UR5Motor are the only Codelets that deals with the environment, closing the sensor-process-act idea.

Finally, we have the ArmAdaptation codelet, representing the Adaptation Codelet in this controller. It is this codelet's responsibility to do any major modification regarding the structure of the mind, including add and kill codelets in execution time, which means, while the agent is running, "alive". Here, after the training processes, it will kill all Behavioral Codelets except for the best one, ending the behavior model learning. This doesn't means a permanent stop from learning, but it ends a MODEL search. This is in accordance with neuron loss on humans.

In addition to these structures, despite not been directly part of the controller, there are also five ROS Nodes (Talker, TimeListener, JointListener, ConnectorPositionListener, ConnectorOrientationListener). The purpose of ROS will be discussed in section 3.1.

\subsection{The Cognitive Cycle}

Most of the cognitive cycle here is very simple. Data is acquired from the environment by the VrepSensor and sent to the Perceptual Codelets. Then, the SelfPerceptual codelet stores information until reaching 50 entries, moment when it will pass them to the Behavioral Codelets. Meanwhile, the TaskPerceptual codelet checks if the current target was accomplished (removing it from the list if so), updates the current target and turns a flag that signalize which behavioral codelet will act or if the Adaptation Codelet should act.

This turn scheme is also simple: once per turn, one behavioral codelet will be active at full strength (activation value 1), the rest remaining inactive. At the end of the round, if the training part is complete, the ArmAdaptation codelet comes in play, as explained in the previous section.
The only active Behavioral Codelet passes then its instructions to the UR5Motor codelet, which directs it to the environment.

\section{EXPERIMENTS}

\subsection{Involved Softwares}

Besides the controller itself, which was coded in Java, we used the V-REP virtual environment (Rohmer et al., 2013), alongside ROS Melodic (Quigley et al., 2009) to communicate with it, all of this running inside a Docker ${ }^{1}$ container to minimize the machine-specific issues that could arise.

$\mathrm{V}$-REP is a general-purpose robotic simulation framework aimed at turning simulation and simulation models more accessible to the general public by offering vast built-in functionalities (and models). The environment also allows experimentation with a variety of control techniques. This environment has plugins to some of the mainstream protocols used in robotics, including ROS. Here, we used a UR5 arm built-in model, a representation (with involved physics) of the Universal Robots ${ }^{2}$ 6-degree-of-freedom arm with the same name, to control directly the six joints.

ROS (Quigley et al., 2009) is an open-source software that provides a structured layer with communications with the purpose of controlling robots. This layer sits upon the host operational systems, even in an heterogeneous cluster. Here, we used both the original ROS and the ROSJAVA version to create the communication structure needed to the experiments. Basically, the structure hangs on the existence of a central node, the roscore. Once this node is up, it is responsible to correctly connect other created nodes. The communication itself is given by a PublisherSubscriber logic, where a node either writes on (publishes) or reads from (subscribes) a specific topic.

The final main software used here is Docker. Docker is a software that combines containers and OS virtualization to create isolated, consistent environment that may be used across a variety of machines and yet have almost the same configurations. This solves various problems when dealing with scientific simulations. Reproducibility, dependency management and version management are some of the issues solved by the use of such strategy, as discussed by Boettiger (2015).

\subsection{Methodology}

In order to evaluate the behaviors' performance, and controller's along, we rely on both summed squared error (L2) and root mean squared error (rmse). The prior was used as loss function in the networks training, i.e., to decide whether the networks is good enough or not, while the second was used when the ArmAdaptation codelet had to decide for the best behavior.

Even having explicit error values, a concept of target accomplishment was used in order to have a more concrete idea of performance. An accomplishment is true when the following conditions are true:

\footnotetext{
1 http://www.docker.com/

2 https://www.universal-robots.com/products/ur5-robot/
} 


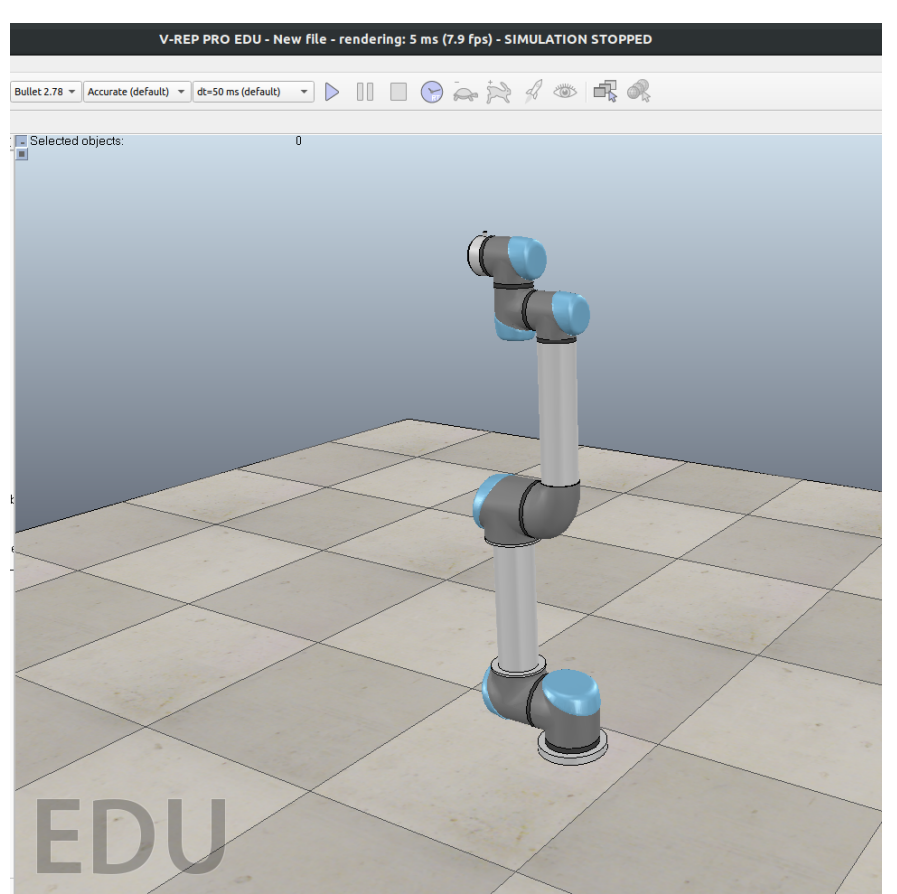

Figure 4. Screenshot of the V-REP environment with the UR5 model on it.

- the connector current position, relative to the first joint, is at maximum 0.02 meters away from target position

- the connector current angles are not more than 0.1 radians each away from the target orientation.

If at least $50 \%$ of the tasks were accomplished, them the experiment was considered a success.

\subsection{Experiments}

To test the controller, and thus the proposed approach, we used a UR5 robotic arm model on the V-REP environment with the ROS protocol (Melodic version) running inside a Docker container to minimize machine-specific issues, as already discussed. The docker image used in this experiments has the following relevant information:

- Ubuntu 16.04 Operational System

- Java 10 (openjdk 10.0.2 2018-07-17)

- ROS Melodic Desktop (ros-melodic-desktop=1.4.1$\left.0^{*}\right)$

- V-REP PRO EDU V3.5.0 (for Ubuntu 16.04)

Each Behavioral Codelet was generated as an instance of a Java class (BasicBehavior) that takes a 4 elements list to mount a neural network with the correspondent number of neurons in each respective layer. So, for example, a $6,20,10,6$ entry would make a network with 6 neurons on the input and output layers and 20 and 10 in the hidden layers, in this order. The input and output layers have a fixed number of neurons, as they represent target connector pose (position and orientation) and joints positions, respectively. The other two numbers were generated with a fixed value, plus a uniformly distributed random integer, as follows:

$$
n=25+X \sim U(0,10)
$$

In the experiments we generated 3 behaviors in this fashion.

As a way to explore on the environment and get enough information to train the networks a total of 50 target poses were randomly generated taking in account the workspace of the arms, shown in figure 3. Initially, the joints positions were unpredictable and made the connector go nowhere near the original poses, but the data gathered from this exploration was enough to train the networks to correctly achieve poses in the space of the visited areas.

In addition to the information already given about the network configuration (see section 2), it's also relevant to say that an early stop method with 3000 maximum iterations was used, that is, the returned result is the best network configuration among the 3000 epochs.

After the training of all Behaviors, the Adaptation Codelet kills all of them, except the best one and, since the network can only make good approximations inside the region of the space where the arm has explored, another 50 target poses inside this region was generated in order to evaluate the already discussed task accomplishments.

\section{RESULTS}

Table 1. Error Statistics

\begin{tabular}{|c|cccc|}
\hline Error & MSE & MAE & RMSE & RSE \\
\hline col_0 & $6.62828 \mathrm{e}-03$ & $4.69151 \mathrm{e}-02$ & $8.14143 \mathrm{e}-02$ & $2.26834 \mathrm{e}-02$ \\
col11 & $8.03350 \mathrm{e}-03$ & $6.27543 \mathrm{e}-02$ & $8.96298 \mathrm{e}-02$ & $2.04189 \mathrm{e}-02$ \\
col_2 & $1.37811 \mathrm{e}-02$ & $8.69378 \mathrm{e}-02$ & $1.17393 \mathrm{e}-01$ & $3.24489 \mathrm{e}-02$ \\
col_3 & $5.76582 \mathrm{e}-03$ & $6.19900 \mathrm{e}-02$ & $7.59330 \mathrm{e}-02$ & $4.66317 \mathrm{e}-02$ \\
col_4 & $5.03655 \mathrm{e}-03$ & $4.95133 \mathrm{e}-02$ & $7.09686 \mathrm{e}-02$ & $6.72802 \mathrm{e}-03$ \\
col_5 & $1.06381 \mathrm{e}-02$ & $7.71211 \mathrm{e}-02$ & $1.03141 \mathrm{e}-01$ & $3.30798 \mathrm{e}-02$ \\
\hline
\end{tabular}

A table with the best behavior network errors after training for a typical run is presented in table 1 . These values were given by the package built-in method (RegressionEvaluation.stats()) for a 6,29,27,6 configuration network and the PC and R2 values, also given by the method, were omitted.

After the training step, and thus the Behavioral Codelets kills, the Controller was capable of achieving at least the minimal fraction of $70 \%$ of tasks in a typical run, after what the program ends. However, the controller performed poorly (between 0.1 and 0.4 meters) when trying to reach a point too much far from the area that it explored. This shows us that the predictive nature of this learning process is sort of step-wise and extrapolations must be taken carefully.

\section{CONCLUSION AND FUTURE WORK}

Although a simple application, this experiment shows us interesting results and a satisfactory performance in a hard task as pursuing a good model to learn a complicated thing like the inverse kinematic of a 6 DOF robotic arm. Even better: the controller used only information regarding its sensors and a "self-imposed" exploration desire, being in accordance with some theories about how living beings learn and shape their behaviors. The proposed approach underlines some characteristics discussed by Vernon et al. (2011) like preexisting and not so useful behaviors, the 
need with interaction with the surround environment to shape them, constructing effectively a representation of how the agent and the world relate. Also, the primitive form of prediction sustained by Gibson (1988) is seen, so the idea of greater learning in early stages of life (Huttenlocher, 1990).

The controller still has some features that can be improved, as better space exploration, other neural network types as concurrent behaviors and some fine adjustments for a better training, but it shows us that we are in the right direction in our research in artificial general intelligence and how to take biological creatures as inspiration for building better agents.

For future works we plan to apply such technique in more challenging scenarios, with more than one type of behavior, continuous learning process and higher level cognitive features.

\section{REFERENCES}

Anderson, J.R. (1989). A theory of the origins of human knowledge. Artificial intelligence, 40(1-3), 313-351.

Anderson, J.R. (2009). How can the human mind occur in the physical universe? Oxford University Press.

Anderson, J.R., Bothell, D., Byrne, M.D., Douglass, S., Lebiere, C., and Qin, Y. (2004). An integrated theory of the mind. Psychological review, 111(4), 1036.

Arkin, R.C., Arkin, R.C., et al. (1998). Behavior-based robotics. MIT press.

Barsalou, L.W. (2010). Grounded cognition: Past, present, and future. Topics in cognitive science, 2(4), 716-724.

Boettiger, C. (2015). An introduction to docker for reproducible research. SIGOPS Oper. Syst. Rev., 49(1), $71-79$

de Gier, M. (2015). Control of a robotic arm: : Application to on-surface 3D-printing. Master's thesis, Delft Center for Systems and Control.

D'Souza, A., Vijayakumar, S., and Schaal, S. (2001). Learning inverse kinematics. In Proceedings 2001 IEEE/RSJ International Conference on Intelligent Robots and Systems. Expanding the Societal Role of Robotics in the the Next Millennium (Cat. No.01CH37180), volume 1, 298-303 vol.1. doi:10.1109/ IROS.2001.973374.

Gärdenfors, P. (2014). The geometry of meaning: Semantics based on conceptual spaces. MIT Press.

Gibaut, W.S.P. (2018). Uma arquitetura cognitiva para o aprendizado instrumental em agentes inteligentes. Ph.D. thesis, DCA-FEEC-UNICAMP.

Gibson, E.J. (1988). Exploratory behavior in the development of perceiving, acting, and the acquiring of knowledge. Annual review of psychology, 39(1), 1-42.

Gudwin, R., Paraense, A., de Paula, S.M., Fróes, E., Gibaut, W., Castro, E., Figueiredo, V., and Raizer, K. (2017). The multipurpose enhanced cognitive architecture (meca). Biologically Inspired Cognitive Architectures, 22, 20-34.

Huttenlocher, P.R. (1990). Morphometric study of human cerebral cortex development. Neuropsychologia, 28(6), $517-527$.

Ivaldi, S., Lyubova, N., Droniou, A., Padois, V., Filliat, D., Oudeyer, P.Y., Sigaud, O., et al. (2014). Object learning through active exploration. IEEE Transactions on Autonomous Mental Development, 6(1), 56-72.

Kotseruba, I., Gonzalez, O.J.A., and Tsotsos, J.K. (2016). A review of 40 years of cognitive architecture research: Focus on perception, attention, learning and applications. arXiv preprint arXiv:1610.08602.

Laird, J.E. (2012). The Soar cognitive architecture. MIT Press.

Langley, P., Laird, J.E., and Rogers, S. (2009). Cognitive architectures: Research issues and challenges. Cognitive Systems Research, 10(2), 141-160.

Newell, A., Rosenbloom, P.S., and Laird, J.E. (1989). Symbolic architectures for cognition. Technical report, DTIC Document.

Newell, A. and Simon, H.A. (1961). Gps, a program that simulates human thought. Technical report, DTIC Document.

Osman, M. (2004). An evaluation of dual-process theories of reasoning. Psychonomic Bulletin \& Review, 11(6), 988-1010. doi:10.3758/BF03196730.

Paraense, A.L., Raizer, K., de Paula, S.M., Rohmer, E., and Gudwin, R.R. (2016). The cognitive systems toolkit and the $\{\mathrm{CST}\}$ reference cognitive architecture. Biologically Inspired Cognitive Architectures, 17, 32 - 48. doi:http://dx.doi.org/10.1016/j.bica.2016. 07.005. URL //www.sciencedirect.com/science/ article/pii/S2212683X1630038X.

Quigley, M., Conley, K., Gerkey, B., Faust, J., Foote, T., Leibs, J., Wheeler, R., and Ng, A.Y. (2009). Ros: an open-source robot operating system. In ICRA workshop on open source software, volume 3, 5. Kobe, Japan.

Rohmer, E., Singh, S.P.N., and Freese, M. (2013). Vrep: a versatile and scalable robot simulation framework. In Proc. of The International Conference on Intelligent Robots and Systems (IROS).

Simon, H.A. and Newell, A. (1971). Human problem solving: The state of the theory in 1970. American Psychologist, 26(2), 145.

Sloman, A. (2002). Architecture-based conceptions of mind. In In the Scope of Logic, Methodology and Philosophy of Science, 403-427. Springer.

Sun, R. (2004). Desiderata for cognitive architectures. Philosophical Psychology, 17(3), 341-373.

Sun, R. (2007). The importance of cognitive architectures: An analysis based on clarion. Journal of Experimental \& Theoretical Artificial Intelligence, 19(2), 159-193.

Vernon, D., Von Hofsten, C., and Fadiga, L. (2011). A roadmap for cognitive development in humanoid robots, volume 11. Springer Science \& Business Media.

Wang, L..T. and Chen, C.C. (1991). A combined optimization method for solving the inverse kinematics problems of mechanical manipulators. IEEE Transactions on Robotics and Automation, 7(4), 489-499. doi:10.1109/ 70.86079 . 